\title{
Dietary Lipids and Incidence of Cerebral Infarction in a Japanese Rural Community
}

\author{
Fukue Seino, ${ }^{1}$ Chigusa Date, ${ }^{2}$ Takeo Nakayama, ${ }^{1}$ Nobuo Yoshirke, ${ }^{3}$ \\ Tetsuji Yokoyama, ${ }^{1}$ Momoko Yamaguchi, ${ }^{3}$ \\ and Heizo TANAKA ${ }^{1}$ \\ ${ }^{1}$ Department of Epidemiology, Medical Research Institute, Tokyo Medical \\ and Dental University, Chiyoda-ku, Tokyo 101, Japan \\ ${ }^{2}$ Department of Public Health, Osaka City University Medical School, \\ Osaka 545, Japan \\ ${ }^{3}$ Division of Adult Health Science, National Institute of Health \\ and Nutrition, Tokyo 162, Japan
}

(Received August 15, 1996)

Summary Objective: To assess the relationship between dietary lipids and incidence of cerebral infarction in a Japanese rural population. Design: A cohort study from July 1977 through December 1992. Setting: Akadani-Ijimino (A-I) district, Niigata Prefecture, Japan. Subjects and methods: All the residents, 1,182 men and 1,469 women, aged 40 years and over. Out of these members, 954 men and 1,329 women who were initially free of stroke completed a semiquantitative food frequency questionnaire in 1977, and were then subjected to a follow-up for 15.5 years. The occurrence of stroke was determined by the annual follow-up examination and registry. Dietary lipid was adjusted for total energy or fat intake by the residual method. Sex- and age-stratified and blood pressureand atrial fibrillation-adjusted relative risk for cerebral infarction was estimated by the Cox proportional hazard model. Results: There were 75 new cases of cerebral infarction during the observation period. The relative risk for cerebral infarction was less than one in the highest quartile level of total fat, saturated fatty acids (S), Keys score and westernized dietary pattern: $0.68-0.94$. It ranged between 1.36 and 1.57 in the highest level of polyunsaturated (P), n-3 and $n-6$ fatty acids, and P/S ratio. Conclusion: This study suggests the possibility that the traditional Japanese diet, very low fat intake, was likely to increase the risk of stroke through the low level of serum cholesterol as an intermediary factor.

Key Words dietary lipids, cerebral infarction, cohort study, serum cholesterol, "diet-stroke" hypothesis

According to the "diet-heart" hypothesis $(1,2)$, a high intake of saturated fatty acids and cholesterol and low intake of polyunsaturated fatty acids increase the 
level of serum cholesterol, and hypercholesterolemia results in the occurrence of coronary heart disease and stroke. In Japan, however, some ecological studies compared stroke incidence rates among several defined populations, and showed that the rates were inversely correlated with mean serum cholesterol and fat intake $(3,4)$. Afterwards, some cohort studies indicated that the level of serum cholesterol was inversely associated with the risk of stroke; particularly cerebral hemorrhage and cerebral infarction in perforating artery regions after adjustment for some confounding variables such as sex, age and blood pressure (5-7). To date, however, there have been few prospective studies on the relationship between dietary fat and stroke among Japanese in Japan. Using all of the residents aged 40 years and over in Akadani-Ijimino district (hereinafter referred to as A-I district), Shibata City, Niigata Prefecture, Japan, their dietary lipid intakes were assessed by a semiquantitative food frequency questionnaire in July 1977. Those who were initially free of stroke have been subjected to a follow-up ever since. In this report, we deal with the relationship of dietary lipid intake to the subsequent 15.5-year incidence of cerebral infarction which shows the highest incidence among the subtypes of stroke.

\section{METHODS}

Subjects. The Shibata Study is a longitudinal study of cardiovascular diseases in Japan. Using all of the residents aged 40 years and over, 1,182 men and 1,469 women, as the eligible population, a baseline examination was conducted in July 1977. The details of the method have already been described in previous reports $(8,9)$. Of the items examined at entry, an epidemiological analysis was performed on three risk factors for this report: blood pressure, atrial fibrillation on electrocardiogram and serum total cholesterol.

We excluded the subjects ( 7 men and 12 women) who could not complete a dietary interview because of hearing impairment or refusal from this analysis. We also excluded the subjects ( 39 men and 20 women) who had a previous onset of stroke before the baseline examination. We followed the remaining 954 men and 1,329 women during the subsequent 15.5 years.

Dietary assessment. To assess habitual dietary intake, the average intake over a one-year period, we developed a validated semiquantitative food frequency questionnaire (FFQ) (10). A long list of foods was derived from a table of Japanese food composition (11) and systematically reduced with the help of an experienced staff dietitian. Finally, the FFQ consisted of 66 food items. In addition, it solicited information on the amount of salt, sugar and oil which were used for flavoring in the A-I district. As for steamed rice, which is consumed at almost every meal by Japanese, we asked the subjects how many bowls of rice they took per day and how large the bowl was: small, medium or large. Trained dietitians asked the participants the frequency of consumption of a given unit or portion size for each of the foods. The response categories to frequency were as 
follows: almost never, 1-2 times, 3-5 times or 6-7 times per week. Food models, household measures and food photograph booklets were used to determine portion sizes. Total intake of a nutrient was the sum of the products of frequency weight and the nutrient content for each food item (i.e., $\Sigma$ (frequency weight $\times$ nutrient content)). Sources of nutrient composition data were based on the Standard Table of Food Composition in Japan and others $(12,13)$.

The validity of the FFQ to discriminate among individuals was assessed by a comparison with individual estimates of nutrient intake measured by the diet records for which foods were weighed among 33 men and women (14). Each participant was instructed by a research dietitian in weighing and recording food intake for one week. The process of keeping a one-week diet record was repeated at one-month intervals over a one-year period (i.e., for a total of 12 weeks). At the end of the 12th week, the FFQ was completed. The correlation coefficient between the crude intake of energy by FFQ and the mean of 12 one-week diet records was 0.60. The coefficients were 0.48 for total protein, 0.36 for total fat, 0.44 for saturated fatty acids, 0.33 for monounsaturated fatty acids, 0.38 for polyunsaturated fatty acids, 0.34 for $n-3$ fatty acids, 0.39 for $n-6$ fatty acids and 0.61 for Keys' Diet Factor (hereinafter Keys score) (15). A regression model included total energy intake as the independent variable and lipid intake as the dependent variable. After using the residual from the regression model as an adjustment for total energy intake (16), the correlations were improved: $0.51,0.62,0.63,0.57$, $0.54,0.43$ and 0.36 (from total protein to $n-6$ fatty acids).

In addition to the lipid intakes mentioned above, two summary indices of the dietary pattern were employed. One was Keys score. The other was extracted from the principal component analysis to which the correlation matrix among 22 food groups was subjected: rice, bread, noodles, fat and oil, sugar, milk and dairy products, eggs, meat except fish, fish, processed fish, salted vegetables, vegetables, potatoes, soybean paste, soybean products, seaweeds, salt for seasoning, fruits, nonalcoholic beverages, alcoholic beverages, Japanese-style confectioneries and western-style confectioneries. Among seven principal components with an eigenvalue more than 1 , the first component (eigenvalue $=2.34$ ), named as a factor of "westernized diet" according to the factor loading matrix, was used in this study.

Follow-up and identification of stroke. The occurrence of stroke in the cohort was determined by both annual follow-up examination and registry. The follow-up examination was the means of detecting patients who survived stroke but who were not reported through the system below. A registration system which covered the whole city of Shibata was incorporated with the local administration and medical association. The sources of notification of possible stroke patients to the registry were general practitioners, public health nurses and ambulance personnel. The patients were also referred by lay personnel in change of health services in the A-I district. Death certificates, social insurance records and medical records of the clinics and hospitals were reviewed periodically. Of the patients identified as such, clinical information was collected from subjects themselves if possible, their rela- 
tives and attending physicians. All information comprising signs and symptoms at the time of the attack, results of the physical examination, laboratory findings and results of special procedures including computed tomography (CT) and cerebral angiography was assessed jointly by three physicians to determine if the case met the criteria for stroke.

Stroke in this study refers to a case where a rapidly developing focal disturbance (or global disturbance in the case of deep coma and subarachnoid hemorrhage) of the brain due to vascular lesion and its signs and symptoms lasted more than $24 \mathrm{~h}$ or until death. Clinically, it included cerebral hemorrhage, cerebral infarction and subarachnoid hemorrhage. Transient cerebral ischemic attack was excluded from this definition. The method of the follow-up and the differential diagnosis of the stroke subtype are published elsewhere (8).

Statistical analyses. It is usually desirable in epidemiologic analyses of dietdisease relationships to employ a measure of nutrient intake which is independent of total caloric intake (16). "Energy-adjusted" total fat intake was computed as the residual from a regression model with total caloric intake as the independent variable and absolute total fat intake as the dependent variable. Furthermore, to estimate the specific effect of a type of fatty acid or cholesterol intake independently of total fat intake, a residual was also computed with the fatty acid or cholesterol intake as the dependent variable and energy-adjusted total fat intake as the independent variable (1).

The Cox proportional hazard regression model (17) with a person-year approach (PHREG Procedure, SAS ver. 6.03) was used. The relative risk or hazard ratio of cerebral infarction was estimated for each of the dietary intakes, which was divided into four categories by quartile (i.e., Q1, Q2, Q3 and Q4) in the order from the bottom level upward. First, sex- and age-stratified relative risk $\left(R_{1}\right)$ was computed by the model including energy, energy-adjusted total fat and total fat-adjusted intake of a type of lipid. The latter term would indicate whether it was uniquely associated with cerebral infarction apart from its contribution to total energy and overall fat intake. Second, diastolic blood pressure (DBP) and atrial fibrillation (AF), which appeared to be statistically significant risk factors for cerebral infarction in our previous report (9), were entered as other covariates in the model, and the second relative risk $\left(R_{2}\right)$ was computed.

Finally, in the stepwise Cox proportional hazard regression model, sex and age were stratified, and DBP, AF, energy intake, energy-adjusted total fat intake, $\mathbf{P} / \mathbf{S}$ ratio, Keys score, the summary index (the factor score of the first principal component) and fat-adjusted intakes of six types of lipid (13 variables in total) were entered as independent variables in the model. Statistically significant factors were selected at the level of $p<0.1$ by the stepwise procedure. 


\section{RESULTS}

\section{Incidence}

During the 15.5 years of observation, 234 men and 226 women died from all causes, and 38 men and 85 women were lost due to moving. There were 141 new cases of stroke between July 1977 and December 1992. By subclassification, 75 were cerebral infarction, 27 were cerebral hemorrhage, 11 were subarchnoid hemorrhage and 28 were an undetermined type of stroke. Computed tomographic (CT) findings of cerebral infarction were available for 43 cases (nine for cerebral thrombosis in cortical artery regions, 16 for cerebral embolism, 16 for cerebral infarction in penetrating artery regions and two in others), most of which occurred during the second half of the observation period when CT scanners became available in some hospitals in the Shibata area.

\section{Baseline characteristics of the subjects}

The levels of systolic and diastolic blood pressure and serum total cholesterol and the number of cases with AF on ECG at the baseline examination are shown by sex and age in Table 1. The daily intakes of energy and lipids are also presented in Table 1. The highest average intake of total fat was $52.9 \mathrm{~g} / \mathrm{day}$, which was observed in the age group of men $40-49$ years, and the $\mathrm{P} / \mathrm{S}$ ratio was more than 1.0 in any age group. Thus, lipid intakes in the A-I district were quite different from those in west European and American countries, not only in quantity but also in quality.

\section{Dietary lipids and serum cholesterol}

The partial correlation coefficients were computed between serum total cholesterol and dietary lipids at baseline, and adjusted for age, sex, energy and/or total fat (Table 2). The intakes of energy, total fat (energy-adjusted), saturated fatty acids (SFA, total fat-adjusted), monounsaturated fatty acids (MUFA, total fat-adjusted) and the summary index of the dietary pattern (westernized pattern of diet) were significantly correlated to the level of serum cholesterol, although the strength of association was weak.

\section{Dietary lipids and cerebral infarction}

In Table 3, the relative risks or hazard ratios for cerebral infarction by quartile of the dietary intake are shown when the risk in the lowest quartile level, Q1, is 1.00 (the reference). Energy intake had a tendency to be inversely related to the risk of cerebral infarction, but the trend was not statistically significant because the highest risk was observed in the $\mathrm{Q} 2$ level. The sex- and age-stratified relative risk $\left(\mathrm{RR}_{1}\right)$ for Q4 was 0.66 (95\% confidence interval: 0.26-1.64), and the sex- and age-stratified relative risk adjusted for DBP and AF on ECG $\left(R_{2}\right)$ was 0.76 (95\% CI: 0.30 1.95). 


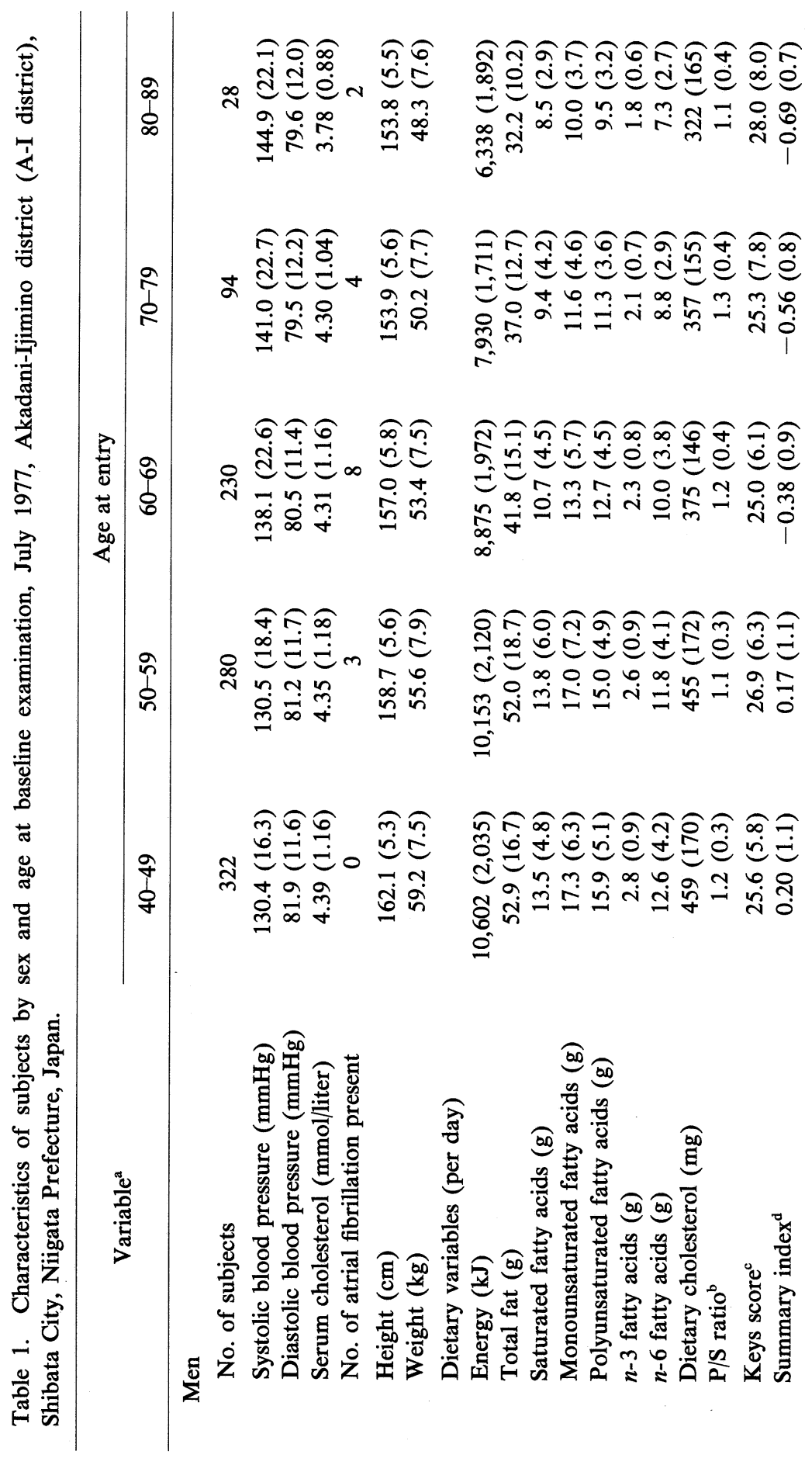




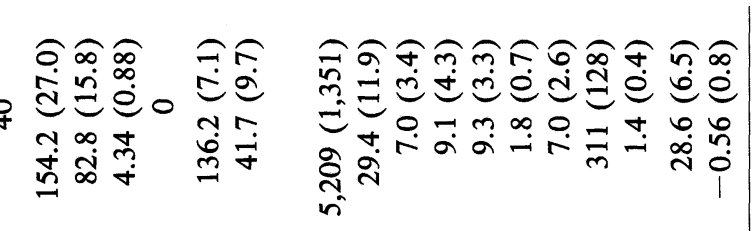

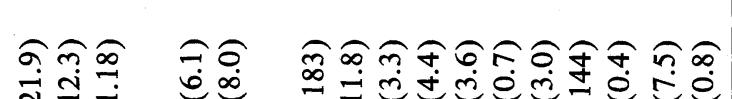

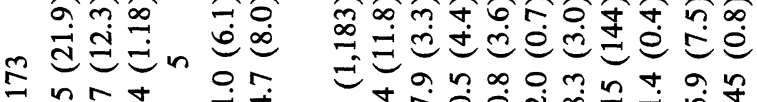

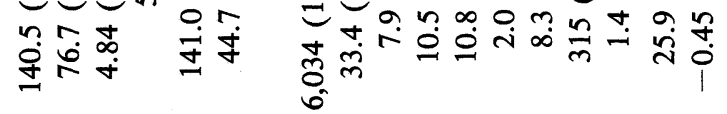

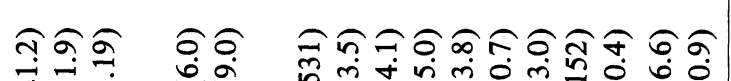

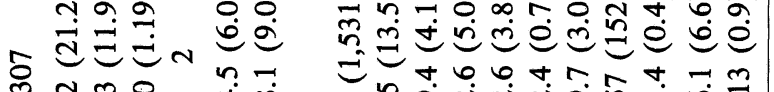

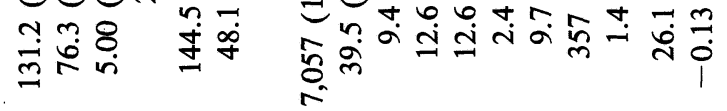

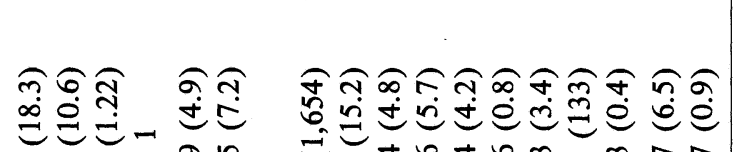

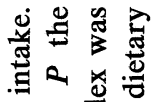

. 0.

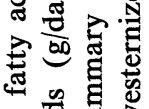

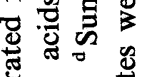

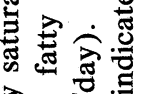

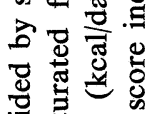

空

新

o nut

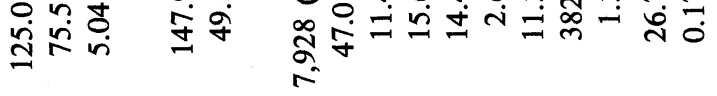

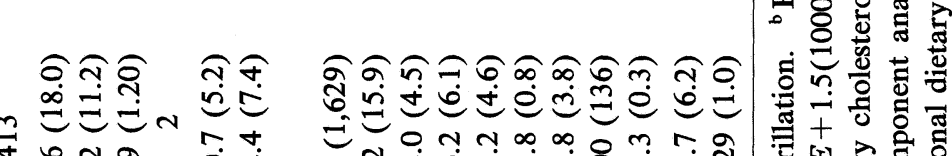

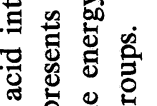

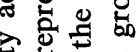

的电

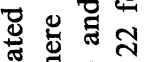

然

勈

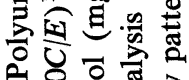

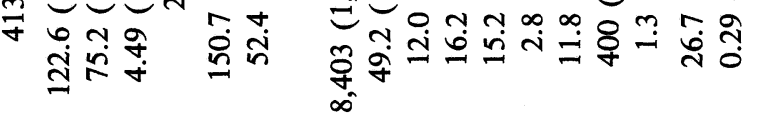

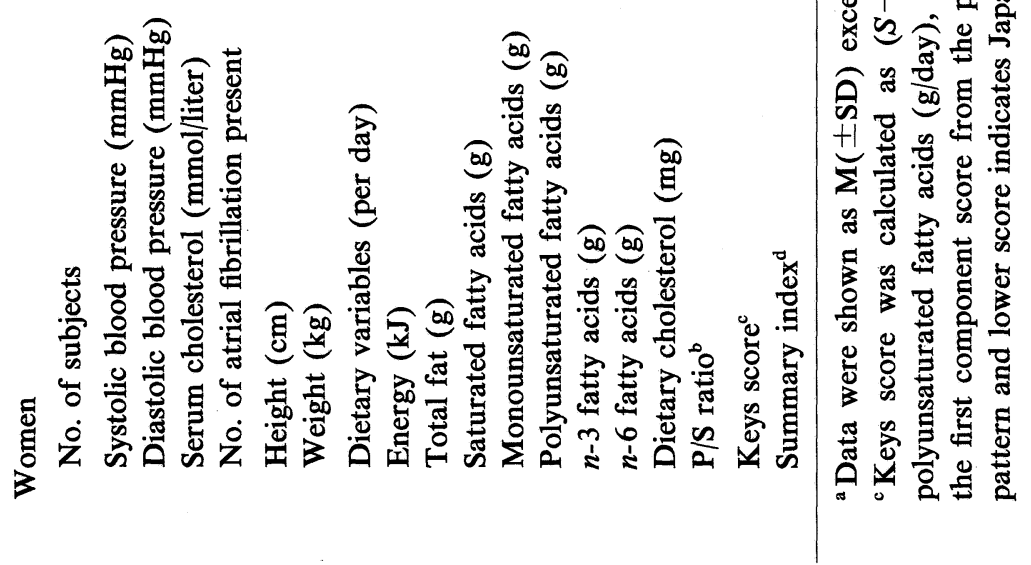

Vol. 43, No. 1, 1997 
Table 2. Partial correlation between serum total cholesterol and dietary lipids at baseline examination, July 1977 (Sex- and age-adjusted, A-I district, Shibata City, Niigata Prefecture, Japan).

\begin{tabular}{|c|c|}
\hline & Serum cholesterol \\
\hline Energy & $0.042^{\mathrm{c}}$ \\
\hline Total fat ${ }^{\mathrm{a}}$ & $0.063^{\mathrm{d}}$ \\
\hline Saturated fatty acids ${ }^{\mathrm{b}}$ & $0.045^{\mathrm{c}}$ \\
\hline Monounsaturated fatty acids ${ }^{b}$ & $0.050^{c}$ \\
\hline Polyunsaturated fatty acids ${ }^{\mathrm{b}}$ & 0.022 \\
\hline$n-3$ fatty acids ${ }^{\mathrm{b}}$ & 0.004 \\
\hline$n-6$ fatty acids ${ }^{b}$ & 0.027 \\
\hline Dietary cholesterol $^{\mathrm{b}}$ & -0.034 \\
\hline $\mathbf{P} / \mathbf{S}$ ratio & -0.031 \\
\hline Keys score & 0.018 \\
\hline Summary index & $0.060^{\mathrm{d}}$ \\
\hline
\end{tabular}

${ }^{a}$ Energy adjusted by the residual method of Willett and Stampfer (1986). ${ }^{\mathrm{b}}$ Adjusted for energy-adjusted total fat by the residual method. ${ }^{\mathrm{c}} p<0.05$. ${ }^{\mathrm{d}} p<0.01$.

A clear relationship between total fat intake and cerebral infarction was not observed, although $R R_{1}$ and $R R_{2}$ for $Q 2, Q 3$ and $Q 4$ were less than one.

As for the various types of lipids, the intake of MUFA was inversely associated and the intakes of PUFA and n-6 FA were positively associated with the development of cerebral infarction, although the relations did not reach statistical significance. There was no overall relationship between SFA, $n-3$ FA, cholesterol or P/S ratio and cerebral infarction, although the lowest risk was observed in the Q4 level of SFA. Keys score was inversely correlated to $R_{2} R_{2}$ for cerebral infarction, although not significant. The lowest risk was seen in the Q4 level of Keys score. As for the summary index, $R_{R}$ and $R_{2}$ in the most westernized pattern of diet, $Q 4$, were lowest, 0.64 and 0.70 respectively.

In the sex- and age-stratified stepwise Cox proportional hazard model (multivariate analysis), no dietary variable was selected as the independent risk factor for cerebral infarction, but the level of diastolic blood pressure and AF on ECG were statistically significant factors.

\section{Serum cholesterol and cerebral infarction}

In the sex- and age-stratified Cox model, the relative risk of cerebral infarction was estimated for the level of serum total cholesterol by an increase of one standard deviation, $1.2 \mathrm{mmol} / \mathrm{liter}$. It was 0.88 (95\% CI: 0.69-1.13).

\section{DISCUSSION}

The inverse relation of the serum level of total cholesterol with the risk of not only cerebral hemorrhage but also cerebral infarction has been reported in numer- 
Table 3. Relative risk (RR) for cerebral infarction by quartile of dietary intake, Cox proportional hazard model, 15.5-year follow-up from July 1977 through December 1992 (A-I district, Shibata City, Niigata Prefecture, Japan).

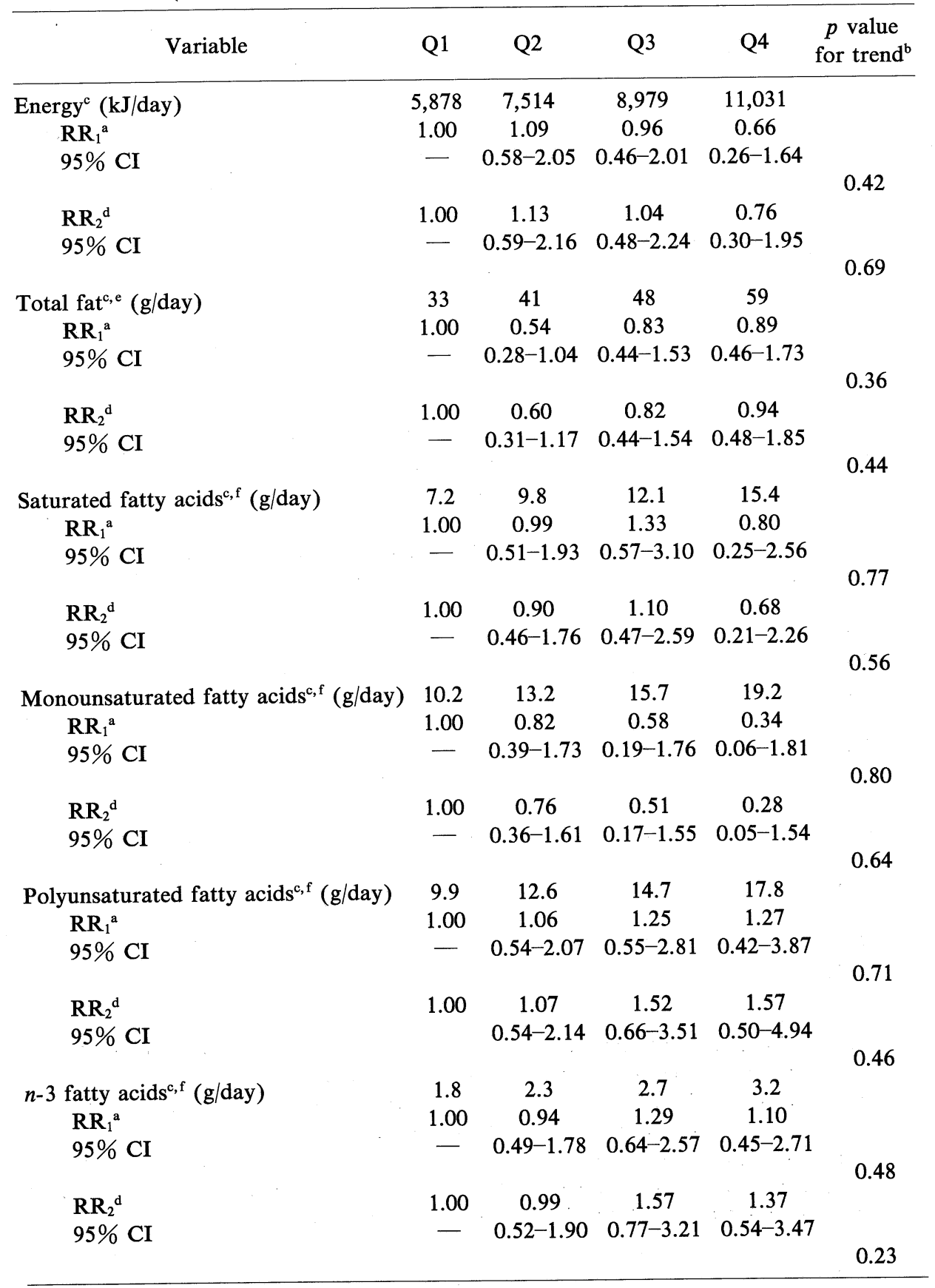

Vol. 43, No. 1, 1997 
Table 3. (continued)

\begin{tabular}{|c|c|c|c|c|c|}
\hline Variable & Q1 & Q2 & Q3 & Q4 & $\begin{array}{c}p \text { value } \\
\text { for trend }{ }^{\mathrm{b}}\end{array}$ \\
\hline$n-6$ fatty acids ${ }^{\mathrm{c}, \mathrm{f}}$ (g/day) & 7.6 & 9.6 & 11.6 & 14.1 & \multirow{5}{*}{0.88} \\
\hline $\mathbf{R R}_{1}{ }^{a}$ & 1.00 & 1.04 & 1.13 & 1.29 & \\
\hline $95 \% \mathrm{CI}$ & - & $0.55-1.98$ & $0.50-2.56$ & $0.45-3.70$ & \\
\hline $\mathbf{R R}_{2}{ }^{\mathrm{d}}$ & 1.00 & 1.07 & 1.31 & 1.54 & \\
\hline $95 \% \mathrm{CI}$ & - & $0.56-2.05$ & $0.57-3.01$ & $0.52-4.56$ & \\
\hline & & & & & \multirow[t]{4}{*}{0.71} \\
\hline Dietary cholesterol $^{\mathrm{c}, \mathrm{f}}(\mathrm{mg} /$ day $)$ & 246 & 341 & 416 & 548 & \\
\hline $\mathbf{R R}_{1}{ }^{\mathrm{a}}$ & 1.00 & 1.21 & 1.46 & 1.03 & \\
\hline $95 \% \mathrm{CI}$ & - & $0.64-2.30$ & $0.76-2.80$ & $0.45-2.38$ & \\
\hline $\mathrm{RR}_{3}^{\mathrm{d}}$ & 100 & 122 & 136 & 1.11 & \multirow[t]{2}{*}{0.63} \\
\hline $95 \% \mathrm{CI}$ & - & $0.64-2.31$ & $0.70-2.62$ & $0.48-2.56$ & \\
\hline & & & & & \multirow[t]{4}{*}{0.58} \\
\hline $\mathbf{P} / \mathbf{S}$ ratio $^{c}$ & 0.9 & 1.1 & 1.4 & 1.7 & \\
\hline $\mathbf{R R}_{1}{ }^{\mathrm{a}}$ & 1.00 & 0.84 & 1.66 & 1.24 & \\
\hline $95 \% \mathrm{CI}$ & - & $0.39-1.81$ & $0.86-3.18$ & $0.64-2.43$ & \\
\hline & & & & & \multirow[t]{3}{*}{0.65} \\
\hline $\mathbf{R R}_{2}{ }^{\mathrm{d}}$ & 1.00 & 0.85 & 1.62 & 1.36 & \\
\hline $95 \% \mathrm{CI}$ & - & $0.40-1.83$ & $0.84-3.12$ & $0.70-2.68$ & \\
\hline Keys score ${ }^{c}$ & 20 & 24 & 28 & 34 & \multirow{3}{*}{0.49} \\
\hline $\mathbf{R R}_{1}{ }^{\mathrm{a}}$ & 1.00 & 0.82 & 0.84 & 0.79 & \\
\hline $95 \% \mathrm{CI}$ & - & $0.44-1.54$ & $0.45-1.57$ & $0.42-1.48$ & \\
\hline & & & & & \multirow[t]{2}{*}{0.62} \\
\hline $\mathbf{R R}_{2}{ }^{\mathrm{d}}$ & 1.00 & 0.94 & 0.89 & 0.79 & \\
\hline $95 \% \mathrm{CI}$ & - & $0.50-1.78$ & $0.48-1.68$ & $0.41-1.49$ & \multirow{2}{*}{0.61} \\
\hline Summary index ${ }^{c}$ & -1.06 & -0.40 & 0.23 & 1.12 & \\
\hline $\mathrm{RR}_{1} \mathrm{a}^{\mathrm{a}}$ & 1.00 & 0.65 & 0.75 & 0.64 & \\
\hline $95 \% \mathrm{CI}$ & - & $0.36-1.17$ & $0.41-1.37$ & $0.31-1.32$ & \multirow[b]{2}{*}{0.15} \\
\hline $\mathrm{RR}_{2}{ }^{\mathrm{d}}$ & 1.00 & $\begin{array}{c}0.68 \\
037-1\end{array}$ & $\begin{array}{c}0.79 \\
0.15\end{array}$ & $\begin{array}{c}0.70 \\
0.32-1.45\end{array}$ & \\
\hline $95 \%$ CI & - & $0.37-1.22$ & $0.43-1.45$ & $0.33-1.45$ & 0.29 \\
\hline
\end{tabular}

${ }^{\text {a }}$ Sex- and age-stratified relative risk or hazard ratio by Cox proportional hazard model. ${ }^{\mathrm{b}}$ Trend was calculated using dietary factors as continuous variables. ${ }^{\mathrm{c}}$ Median intake for the quartile. ${ }^{\mathrm{d}}$ Relative risk after sex and age were adjusted, and diastolic blood pressure and atrial fibrillation were adjusted by the Cox proportional hazard model. ${ }^{\mathrm{e}}$ Total energy-adjusted intake of total fat and intake of total energy were entered in the model. ${ }^{\mathrm{f}}$ Total fat adjusted intake of a specific type of lipid, and total energyadjusted intake of total fat and total energy were entered in the model. 
ous studies of Japanese populations which were conducted during the high economic growth period from 1960 to 1975 (5-7). A mechanism, speculatively, involves the weakening of the endothelium of smaller intracerebral arteries due to low serum cholesterol levels (18). This condition may be further aggravated by hypertension and lead to cerebral hemorrhage if severe hypertension exists, or to cerebral infarction in penetrating artery regions if mild to moderate hypertension is sustained. Before and during the high economic growth period, the Japanese adhered to traditional lifestyles, particularly dietary habits characterized by high salt and carbohydrate intake, and low fat and animal protein intake. Thus, this study was planned to test the "diet-stroke-hypothesis": low intake of lipids and low level of serum cholesterol result in the development of stroke. The first aim was to reconfirm the relation of dietary lipids to serum total cholesterol at the baseline examination by a cross-sectional study, and the second was to analyze the relation of the level of serum total cholesterol or dietary intake of various lipids with the risk of cerebral infarction by a cohort study. Data relating specific fatty acids or cholesterol intake to a risk of stroke were expected to provide sound dietary guidance for persons wishing to reduce their risk of stroke.

Since the number of patients with cerebral hemorrhage was relatively small, the relation between diet and cerebral hemorrhage was not analysed in this study.

Dietary lipids and serum cholesterol. Our findings provided general support for the existing studies of dietary lipids and serum total cholesterol levels. However, the correlations were small; as the study population was relatively large, these small correlations were statistically significant. According to Willett (1) and Jacobs et al. (19), the expected correlation between dietary lipids and serum cholesterol in a free-living population would be less than 0.1 , because many factors including genetic and lifestyle determinants influence serum cholesterol. For example, Shekelle et al. (20) observed that the correlation between Keys score and serum total cholesterol was 0.08 in the Western Electric Study.

On the other hand, there was a discrepancy between our results, indicating a positive association of the intake of monounsaturated fatty acids (MUFA) with the serum levels of total cholesterol, and existing reports that MUFA, particulary oleic acid, lower serum cholesterol levels $(21-23)$. The probable explanation is that, in the A-I district, the main food sources of MUFA were eggs, pork, mayonnaise and vegetable oil for flavoring and tempura in common with those of SFA and, therefore, MUFA were strongly correlated with SFA ( $r=0.88$ for the crude fatty acids, $r=0.66$ for the total fat-adjusted fatty acids). The intake of polyunsaturated fatty acids (PUFA) and $n-6$ fatty acids ( $n-6$ FA) also showed a positive, but non-significant, relation to serum cholesterol, although an inverse relation between $\mathbf{P} / \mathbf{S}$ ratio and serum cholesterol was observed. The reason was not clear, but our finding might indicate that the substitution of PUFA or $n-6$ FA for SFA would be more affective for the reduction of serum cholesterol levels than an increase in PUFA or $n-6$ FA independently of SFA. Another reason might be the heterogeneity of PUFA. Not all PUFA have a similar effect on serum cholesterol, and many 
types of PUFA may compete with each other at enzymatic sites (24).

Serum cholesterol and cerebral infarction. A weak and inverse relationship (hazard ratio for $1 \mathrm{SD}=0.88$ ) between serum total cholesterol level and the development of cerebral infarction was seen in this study, but was not statistically significant, while in Japanese studies during the high economic growth period, statistical significance was attained and the hazard ratio was less than $0.88(5-7)$. In the United States, however, a relatively strong and positive relationship between serum cholesterol and cerebral infarction is usually observed $(25,26)$. Possible differences in the effects of serum cholesterol at different vascular sites could lead to the complex association between serum cholesterol levels and cerebral infarction as a single entity. On the basis of the theory of Kuller and Reisler (27) and epidemiological studies in Japan (28), US (29) and Europe $(30,31)$, hypercholesterolemia is assumed to be positively associated with cerebral thrombosis in cortical artery regions, but serum cholesterol levels are inversely related to cerebral infarction in penetrating artery regions. Because cerebral infarction occurs much more frequently in cortical than in penetrating artery regions in the US and Europe, hypercholesterolemia is positively related to cerebral infarction as a single entity. In contrast, cerebral infarction in penetrating artery regions were the most prevalent of infarction subtypes in traditional Japanese. In such a case, serum cholesterol would be inversely related with overall cerebral infarction. The ratio of cerebral thrombosis in cortical artery regions to cerebral infarction in penetrating artery regions would increase with rise in the serum levels of cholesterol in Japanese populations, including the A-I district, during and after the high economic growth period, but the magnitude of the ratio would still be smaller in Japan than in the US and Europe. Thus, a slightly inverse relationship of serum cholesterol level to overall cerebral infarction was observed in this study, but was not statistically significant.

Dietary lipids and cerebral infarction. In contrast to the "diet-heart" hypothesis, the results from the analyses of $R R_{1}$ and $R R_{2}$ (Table 3) for cerebral infarction suggest the possibility that the intake of SFA decreased, and that the $\mathrm{P} / \mathrm{S}$ ratio increased the risk of cerebral infarction through serum total cholesterol as an intermediary factor between dietary lipids and cerebral infarction. The relative risks in the fourth quartile (Q4) of the dietary intake seemed small, but they were consistent with relative risks on the order of 0.7 to 1.5 in most studies of diet and disease (1) and would potentially be important because the dietary exposures are common. In addition, there are other studies which support this "diet-stroke" hypothesis. In the Seven-Country-Study, an ecological study, death rates from cerebrovascular disease were inversely related to the average intake of SFA and MUFA (32). An inverse relation of dietary fat to the subsequent incidence of thrombo-embolic stroke for 10 years was observed in the Honolulu Heart Program (33).

However, both modest relative risks and statistically non-significant findings in this study should be considered here. To examine the validity of our food frequency 
questionnaire (FFQ), we compared the questionnaire with the diet records collected during the preceding year. The correlations ranged from 0.36 for $n-6$ FA to 0.63 for SFA after adjustment for energy or total fat intake by the residual method. The validity of FFQ has been examined in a number of studies. Among them was the validation assessment of a 61-item FFQ used in the Nurses Health Study, in which FFQ scores were compared with the means of four one-week diet records (34). The correlation coefficients were 0.53 for total fat adjusted for energy intake, 0.59 for SFA, 0.48 for PUFA and 0.61 for dietary cholesterol. These figures were almost the same as ours. Therefore, the non-significant $R R_{1}$ and $R_{2}$ could not be explained by the FFQ validity.

The population size and observation period (originally 10 years) in this study were determined according to the incidence of stroke in our previous prospective study (8). In the A-I district, however, decreasing trends in the incidence of stroke were observed, and much more than predicted at the time this study was planned. Thus, an attempt was made to prolong the follow-up period. Nevertheless, sufficient cases of cerebral infarction have not yet been obtained for statistical analyses, as the non-significant findings of $R_{1}$ and $R R_{2}$ suggest. However, the observation period was brought to an end because the period when changes in lifestyle, particularly dietary intake, appeared to end in a relatively stable manner around 1992 in Japan $(35,36)$.

In this study, as in the majority of prospective studies, no attempt was made to take into account possible changes in dietary intake and serum cholesterol levels during the 15.5-year observation period, although dietary intake was unlikely to change significantly as described later.

A significant association was not detectable because some unmeasured third variable was related to diet and cerebral infarction in opposite directions; for example, dietary fiber, exercise, smoking (37) and drinking $(5,38,39)$.

Mertz (40) proposed a hypothetical relationship between the dietary intake factor and health. The dose-response curve was divided into an ascending, horizontal or optimal, and descending segments. If two points (e.g., Q1 and Q4) on the horizontal portion were compared in this study, it might be concluded that the nutrient had no or little effect.

According to the National Nutrition Survey $(35,36,41)$, the intake of fat was very low before the high economic growth period, ranging from $12.0 \mathrm{~g} /$ day in 1946 to $24.7 \mathrm{~g}$ /day in 1960 . Dietary changes were much more marked in the period of high economic growth than in any other period after the Second World War. The increase in fat consumption was the most significant among the major nutrients. The maximum value in this period was $55.2 \mathrm{~g}$ /day (animal fat accounting for 26.2 $\mathrm{g} /$ day) in 1975 . With the oil crisis in 1973 as a turning point, the intake of fat stopped increasing and remained almost unchanged: $58.4 \mathrm{~g} /$ day of total fat $(26.2$ $\mathrm{g}$ /day of animal fat) in 1992. The A-I district was probably following the trend of Japan as a whole, although a few years behind. No information about the approximate latent period between diet and stroke occurrence is available, but the

Vol. 43, No. 1, 1997 
effect of diet would occur many years, a few decades, before the occurrence of stroke. In this study, the baseline intake of lipids was assessed just after the period when the Japanese nutritional status was very poor and, then, it moved rapidly toward western style diet. Therefore, our baseline data might differ from the dietary intakes during the "latent" period, although our FFQ is a method for measuring long-term dietary intake. This would bias relative risks toward the null value of one.

When an inverse relationship between serum cholesterol level and incidence of overall cerebral infarction was clearly observed and the "diet-stroke" hypothesis was formed, the ratio of cerebral thrombosis in cortical artery regions to cerebral infarction in penetrating artery regions was small. As the ratio increased during the observation period, as mentioned earlier (the magnitude of the ratio would still be much smaller in Japan than in the US and Europe), the relative risk for all subtypes of cerebral infarction combined would be attenuated. In this context, the failure to observe a clear association of $n-3$ FA with the risk of cerebral infarction of all subtypes can be explained. From many experimental studies, $n-3$ FA have a variety of physiologic effects which include decreased platelet aggregation and blood viscosity, increased fibrinolytic activity and, perhaps, decreased blood pressure $(24$, 42). These effects would result in a reduction in thrombotic infarction and, therefore, inhibit cerebral thrombosis in cortical artery regions among stroke subtypes.

Antihypertensive medication is an important factor which is closely related to the downward shift in the level of blood pressure and, therefore, in stroke incidence. Twenty-three percent of the initial subjects used antihypertensive medications. To examine the effect of medical treatment on stroke and hypertension, a multivariate analysis for stroke was conducted, including sex, age and mean blood pressure as the independent variables and antihypertensive medication as the dummy variable. Another analysis for systolic blood pressure was done using the interaction variable (43). Neither analysis showed a statistically significant effect of treatment $(p=0.70$ and $p=0.82)$. Thus, it would appear to be valid that we did not take into account antihypertensive medication in this study.

In conclusion, this study supports the "diet-stroke" hypothesis that dietary lipids are likely to be a protective determinant of cerebral infarction, presumably cerebral infarction in penetrating artery regions, taking into account abundant accumulating evidence, although relative risks are modest and do not reach the statistical significance due to several circumstances referred to earlier.

Since CT has made it possible to classify stroke more precisely, it is necessary to separately evaluate the relation of the fractions of dietary lipid to subtypes of stroke in order to understand fully whatever connections may exist between diet and stroke.

Appreciation is extended to Niigata Prefecture Medical Association, Shibata CityToyosaka City-Kitakambara County Medical Association, Niigata Prefectural Shibata 
Hospital and the Department of Health Promotion, City of Shibata. We are indebted to Takashi Yamamoto, Masako Iwaya, Hiroko Baba and all nutritional interviewers and staff who participated in this study.

\section{REFERENCES}

1) Willett, W. (1990): Nutritional Epidemiology, Oxford University Press, New York.

2) Chait, A., Brunzell, J. D., Denke, M. A., Eisenberg, D., Ernst, N. D., Franklin, F. A., Jr., Ginsberg, H., Kotchen, T. A., Kuller, L., Mullis, R. M., Nichaman, M. Z., Nicolosi, R. J., Schaefer, E. J., Stone, N. J., and Weidman, W. H. (1993): Rationale of the diet-heart statement of the American Heart Association. Report of the Nutrition Committee. Circulation, 88, 3008-3029.

3) Komachi, Y., Iida, M., Shimamoto, T., Chikayama, Y., Takahashi, H., Konishi, M., and Tominaga, S. (1971): Geographic and occupational comparison of risk factors in cardiovascular disease in Japan. Jpn. J. Circ., 35, 189-207.

4) Shigiya, K., and Komachi, Y. (1976): Correlation between nutritional status and disease of circulatory system from an epidemiological point of view, in Nutritional Status and Disease of Circulatory System in the Japanese, ed. by Shigiya, K., Komachi, Y., and Watanabe, T., Hoken Dojin Sha, Tokyo, pp. 1-42 (in Japanese).

5) Tanaka, H., Ueda, Y., Hayashi, M., Date, C., Baba, T., Yamashita, H., Shoji, H., Tanaka, Y., Owada, K., and Detels, R. (1982): Risk factors for cerebral hemorrhage and cerebral infarction in a Japanese rural community. Stroke, 13, 62-73.

6) Okada, H., Horibe, H., Ohno, Y., Hayakawa, N., and Aoki, N. (1976): A prospective study of cerebrovascular disease in Japanese rural communities, Akabane and Asahi, Part 1, Evaluation of risk factors in the occurrence of cerebral hemorrhage and thrombosis. Stroke, 7, 599-607.

7) Ueshima, H., Iida, M., Shimamoto, T., Konishi, Y., Tsujioka, K., Tanigaki, M., Nakanishi, N., Ozawa, H., Kojima, S., and Komachi, Y. (1980): Multivariate analysis of risk factors for stroke, eight-year follow-up study of farming village in Akita, Japan. Prev. Med., 9, 722-740.

8) Tanaka, H., Ueda, Y., Date, C., Baba, T., Yamashita, H., Hayashi, M., Shoji, H., Owada, K., Baba, K., Shibuya, M., Kon, T., and Detels, R. (1981): Incidence of stroke in Shibata, Japan: 1976-1978. Stroke, 12, 460-466.

9) Tanaka, H., Hayashi, M., Date, C., Imai, K., Asada, M., Shoji, H., Okazaki, K., Yamamoto, H., Yoshikawa, K., Shimada, T., and Lee, S. I. (1985): Epidemiologic studies of stroke in Shibata, a Japanese provincial city: Report on risk factors for cerebral infarction. Stroke, 16, 773-780.

10) Date, C., Tanaka, H., Hayashi, M., Imai, K., Asada, M., Kurihara, H., and Tsuchida, M. (1985): A 6.5-year follow-up study on the relationship between nutrition and cerebral infarction. Osaka City Med. J., 31, 41-63.

11) Resources Council Science and Technology Agency, Japan (1963): Standard Tables of Food Composition in Japan, 3rd rev. ed., Ministry of Finance Printing Bureau, Tokyo.

12) Resources Council Science and Technology Agency, Japan (1982): Standard Tables of Food Composition in Japan, 4th rev. ed., Ministry of Finance Printing Bureau, Tokyo.

13) Resources Council Science and Technology Agency, Japan (1989): Standard Tables of Food Composition in Japan, 4th rev. ed., Appendix II. Fatty acids, Cholesterol, and 
Vitamin E (Tochopherols), Ministry of Finance Printing Bureau, Tokyo.

14) Date, C., Fukui, M., Shimada, T., Fujii, C., Yanagi, M., Shirota, K., Monna, T., Yoshiike, N., Iwaya, M., Matsumura, Y., Sugiyama, M., Yamaguchi, M., Nakayama, T., Yokoyama, T., Chen, H., Seino, F., Iwaoka, H., Zaman, M. M., Shimozato, M., and Tanaka, H. (1995): Reproducibility and validity of a newly developed food frequency questionnaire. J. Health Welfare Statistics, 42(2), 22-29 (in Japanese).

15) Fetcher, E. S., Foster, N., Anderson, J. T., Grande, F., and Keys, A. (1967): Quantitative estimation of diets to control serum cholesterol. Am. J. Clin. Nutr., 20, 475-492.

16) Willett, W., and Stampfer, M. J. (1986): Total energy intake: Implications for epidemiologic analyses. Am. J. Epidemiol., 124, 17-27.

17) Cox, D. R. (1972): Regression models and life tables. J. Royal Statist. Soc., B.34, 187220.

18) Bronner, L. L., Kanter, D. S., and Manson, J. E. (1995): Primary prevention of stroke. N. Engl. J. Med., 333, 1392-1400.

19) Jacobs, D. R., Anderson, J. T., and Blackburn, H. (1979): Diet and serum cholesterol: Do zero correlations negate the relationship? Am. J. Epidemiol., 110, 77-87.

20) Shekelle, R. B., Shryock, A. M., Paul, O., Lepper, M., Stamler, J., Liu, S., Raynor, W. J., Jr. (1981): Diet, serum cholesterol, and death from coronary heart disease. The Western Electric Study. N. Engl. J. Med., 304, 65-70.

21) Mattson, F. H., and Grundy, S. M. (1985): Comparison of effects of diertary saturated, monounsaturated, and polyunsaturated fatty acids on plasma lipids and lipoproteins in men. J. Lipid Res., 26, 194-202.

22) Grundy, S. M. (1986): Comparison of monounsaturated fatty acids and carbohydrates for lowering plasma cholesterol. N. Engl. J. Med., 314, 745-748.

23) Mensink, R. P., and Katan, M. B. (1989): Effect of dietary monounsaturated or polyunsaturated fatty acids on levels of low-density and high-density lipoprotein cholesterol in healthy men and women. N. Engl. J. Med., 321, 436-441.

24) Ulbricht, T. L. V., and Southgate, D. A. T. (1991): Coronary heart disease: seven dietary factors. Lancet, 338, 985-992.

25) Dawber, T. R. (1980): The Framingham Study, The Epidemiology of Atherosclerotic Disease, Harvard University Press, London, pp. 121-141.

26) Benfante, R., Yano, K., Hwang, L., Curb, J. D., Kagan, A., and Ross, W. (1994): Elevated serum cholesterol is a risk factor for both coronary heart disease and thromboembolic stroke in Hawaiian Japanese men, Implications of shared risk. Stroke, 25, 814-820.

27) Kuller, L., and Reisler, D. (1971): An explanation for variations in distribution of stroke and arteriosclerotic heart disease among populations and racial groups. Am. J. Epidemiol., 93, 1-9.

28) Konishi, M., Iso, H., Komachi, Y., Iida, M., Shimamoto, T., Jacobs, D., Terao, A., Baba, S., Sankai, T., and Ito, M. (1993): Association of serum total cholesterol, different types of stroke, and stenosis distribution of cerebral arteries: The Akita Pathology Study. Stroke, 24, 954-964.

29) Iso, S., Jacob, D., Wentworth, D., Neaton, J., and Cohen, J. (1989): Serum cholesterol levels and six-year mortality from stroke in 350,977 men screened for Multiple Risk Factor Intervention Trial. N. Engl. J. Med., 320, 904-910. 
30) Boysen, G., Nyboe, J., Appleyard, M., Sorensen, P., Boas, J., Sominier, F., Jensen, G., and Schnohr, P. (1988): Stroke incidence and risk factors for stroke in Copenhagen, Denmark. Stroke, 19, 1345-1353.

31) Menotti, A., Lanti, M., Seccarceccia, F., Giampaoli, S., and Dima, F. (1993): Multivariate prediction of the first major cerebrovascular event in an Italian population sample of middle-aged men followed up for 25 years. Stroke, 24, 42-48.

32) Ball, M., and Robertson, I. (1994): Do diet influence stroke incidence? N. Z. Med. J., 107, 395-396.

33) Kagan, A., Popper, J. S., Rhoads, G. G., and Yano, K. (1985): Dietary and other risk factors for stroke in Hawaiian Japanese men. Stroke, 16, 390-396.

34) Willett, W. C., Sampson, L., Stampher, M. J., Rosner, B., Bain, C., Witschi, J., Hennekens, C. H., and Speizer, F. E. (1985): Reproducibility and validity of a semiquantitative food frequency questionnaire. Am. J. Epidemiol., 122, 51-65.

35) Tanaka, H., Yamaguchi, M., Date, C., Nakayama, T., Yamamoto, T., Yoshi-ike, N., Iwaya, M., Matsumura, Y., Yokoyama, T., Noji, A., Kushiro, W., and Cho, B. M. (1992): Nutrition and cardiovascular disease, A brief review of epidemiological studies in Japan. Nutr. Health, 8, 107-123.

36) Yamaguchi, M., Yoshi-ike, N., Iwaya, M., and Tanaka, H. (1992): National nutrition survey in Japan. J. Epidemiol., 2 (Suppl.), S53-S62.

37) Shinton, R., and Beevers, G. (1989): Meta-analysis of relation between cigarette smoking and stroke. Br. Med. J., 298, 789-794.

38) Iso, H., Kitamura, A., Shimamoto, T., Sankai, T., Naito, Y., Sato, S., Kiyama, M., Iida, M., and Komachi, Y. (1995): Alcohol intake and the risk of cardiovascular diseases in middle-aged Japanese men. Stroke, 26, 767-773.

39) Kiyohara, Y., Kato, I., Iwamoto, H., Nakayama, K., and Fujishima, M. (1995): The impact of alcohol and hypertension on stroke incidence in a general Japanese population: the Hisayama study. Stroke, 26, 368-372.

40) Mertz, W. (1981): The essential trace elements. Science, 213, 1332-1338.

41) Ministry of Health and Welfare, Japan (1995): National Nutrition Survey 1993. Daiichi Shuppan Publisher, Tokyo (in Japanese).

42) Morris, M. C., Sacks, F., and Rosner, B. (1993): Does fish oil lower blood pressure? A meta-analysis of controlled trials. Circulation, 88, 523-533.

43) Nakayama, T., Date, C., Yokoyama, T., Yoshiike, N., Yamaguchi, M., Tanaka, H. (1997): A 15.5-year follow up study of stroke in a Japanese provincial city: The Shibata Study. Stroke, 28, 45-52. 